\title{
Modelagem de um mancal hidrodinâmico pelo Método dos Elementos de Contorno
}

\author{
Loeffler, C.F. ; Mansur, W.J. ${ }^{2}$
}

1 Programa de Pós-Graduação em Engenharia Mecânica, Universidade Federal do Espírito Santo, Vitória, ES, Brasil 2 Programa de Pós-Graduação em Engenharia Civil, Universidade Federal do Rio de Janeiro, RJ, Brasil

\begin{abstract}
Resumo
Duas formulações alternativas do Método dos Elementos de Contorno são empregadas para modelar e resolver a distribuição do campo de pressões gerado pela hidrodinâmica de um mancal de deslizamento, cuja equação diferencial parcial de governo possui coeficientes variáveis. Dois problemas unidimensionais que dispõem de solução analítica são apresentados com o propósito de validar a consistência da técnica proposta, embora a metodologia seja igualmente aplicável para problemas bidimensionais.
\end{abstract}

\begin{abstract}
Two alternative formulations of the Boundary Element Method are used to model and solve the distribution of the pressure field generated by the hydrodynamic in a journal bearing, a problem in which its governing partial differential equation has variable coefficients. Two one-dimensional problems that have analytical solution are presented in order to validate the proposed technique, although the method can be equally applicable to two-dimensional problems.
\end{abstract}

Palavras chave: Método dos Elementos de Contorno, Mancais de Deslizamento, Funções de Base Radial.

\section{Introdução}

O Método dos Elementos de Contorno (MEC) é uma técnica numérica que vem conquistando interesse cada vez maior no meio acadêmico e industrial, pois apresenta vantagens no trato de problemas com fronteiras móveis, concentração de tensões, fratura, contato, meios infinitos e semi-infinitos e outros casos em que as técnicas de domínio não são adequadas, nem simples de empregar. Entretanto, não é muito versátil na solução de problemas que incluem fontes, sorvedouros, forças de inércia e outras ações domínio similares. Estas deficiências têm sido superadas através de novas estratégias, como a formulação que faz a integração direta no contorno das ações de domínio generalizadas (MECID), que foi aplicada com êxito em problemas governados pela Equação de Poisson [1] e pela Equação de Helmholtz [2].

Neste trabalho, aplica-se a MECID num problema relativamente complexo, referente à distribuição estacionária de pressão ao longo da superfície de contato de um mancal hidrodinâmico. Pode-se verificar a equação de governo deste problema é similar a que descreve potenciais escalares em meios heterogêneos, o que credencia sua aplicação futura em importantes problemas relacionados à sísmica de prospecção, no caso em que as forças de domínio são associadas à inércia do meio físico constituinte.

\section{Equação de Governo}

Mediante uma série de hipóteses simplificadoras, deduz-se a equação diferencial de equilíbrio para o mancal hidrodinâmico que, em condições de unidimensionalidade, é dada por [3]:

$$
\frac{\mathrm{d}^{2} \mathrm{p}}{\mathrm{dx}^{2}}+\frac{3}{\mathrm{~h}(\mathrm{x})} \frac{\mathrm{d}[\mathrm{h}(\mathrm{x})]}{\mathrm{dx}} \frac{\mathrm{dp}}{\mathrm{dx}}=-\frac{\mu}{\left[\mathrm{h}(\mathrm{x})^{3}\right]} \mathrm{V} \frac{\mathrm{d}[\mathrm{h}(\mathrm{x})]}{\mathrm{dx}}
$$

Na equação anterior $\mathrm{p}(\mathrm{x})$ é a sobrepressão com relação à pressão inicial de injeção do óleo, $V$ é uma velocidade média e $\mu$ é a viscosidade. A variável $h(x)$ depende da forma das superfícies examinadas, mas é conhecida, vide figura 1. Impõem-se condições essenciais em termos da pressão $p(x)$ e condições naturais em termos do fluxo $q(x)$ :

$$
\mathrm{q}(\mathrm{x})=\rho \mathrm{h}(\mathrm{x}) \mathrm{v}(\mathrm{x})
$$

$\mathrm{Na}$ expressão do fluxo, $\rho$ é a massa específica e a velocidade $\mathrm{v}(\mathrm{x})$ do lubrificante é dada por: 


$$
\mathrm{v}(\mathrm{x})=\mathrm{V}-\frac{\mathrm{h}(\mathrm{x})^{2}}{\mu} \frac{\mathrm{dh}(\mathrm{x})}{\mathrm{dx}} \frac{\partial \mathrm{p}(\mathrm{x})}{\partial \mathrm{x}}
$$

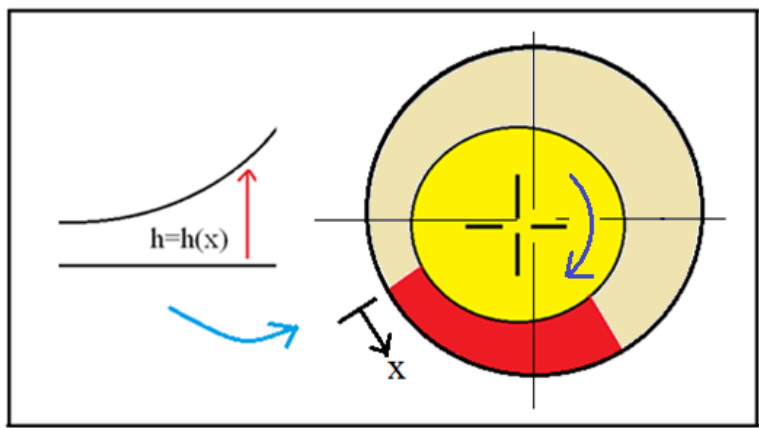

Figura 1: Esquema ilustrativo do modelo do mancal hidrodinâmico, enfatizando o significado da altura $\mathrm{h}(\mathrm{x})$.

\section{Forma Integral Inversa}

Por conveniência, reescreve-se a equação (1):

$$
\left[\mathrm{K}(\mathrm{x}) \mathrm{u}(\mathrm{x}),{ }_{\mathrm{x}}\right],_{\mathrm{x}}=\mathrm{f}(\mathrm{x})
$$

Onde $\mathrm{K}(\mathrm{x})$ representa o conjunto de propriedades do meio físico e $f(x)$ a ação de domínio generalizada. Através do uso da solução fundamental $u^{*}(\xi: x)$ do problema de Poisson [4] e de sua derivada normal $\mathrm{q}^{*}(\xi: \mathrm{x})$, além de recursos matemáticos oriundos da teoria das equações integrais, é possível escrever o lado esquerdo da equação (4) na seguinte forma:

$$
\begin{aligned}
& \int_{\Gamma}\{[\mathrm{K}(\mathrm{x}) \mathrm{q}(\mathrm{x}) \mathrm{u} *(\xi ; \mathrm{x})]-[\mathrm{u}(\mathrm{X}) \mathrm{K}(\mathrm{x}) \mathrm{q} *(\xi ; \mathrm{x})]\} \mathrm{d} \Gamma+ \\
& \int_{\Omega}\left[\mathrm{u}(\mathrm{x})\left[\mathrm{K},{ }_{\mathrm{x}}(\mathrm{x}) \mathrm{u} *(\xi ; \mathrm{x}),{ }_{\mathrm{x}}\right] \mathrm{d} \Omega+\mathrm{c}(\xi) \mathrm{K}(\xi) \mathrm{u}(\xi)=\right. \\
& \int_{\Omega} \mathrm{f}(\mathrm{x}) \mathrm{u} *(\xi ; \mathrm{x}) \mathrm{d} \Omega
\end{aligned}
$$

Para transformar a integral de domínio no lado esquerdo desta última equação usa-se a técnica Quase Dual [5]. Para tratar o lado direito da mesma, empregase a formulação MECID [1], do seguinte modo:

$$
\int_{\Omega} \mathrm{f}(\mathrm{x}) \mathrm{u} *(\xi ; \mathrm{x}) \mathrm{d} \Omega={ }^{\xi} \alpha^{\mathrm{j}} \int_{\Gamma} \eta^{\mathrm{j}}(\mathbf{x}) \mathrm{d} \Gamma
$$

\section{Resultados}

Nos dois exemplos apresentados a seguir, resolveu-se um domínio linear de comprimento unitário com pressão de referência nula na entrada e na saída. Duas malhas com 40 e 80 elementos de contorno lineares são usadas para aproximar o campo de pressão e velocidades, enquanto as funções radiais de placa fina são empregadas na interpolação auxiliar tanto da MECID quanto na formulação Quase-Dual.
4.1 Primeiro Exemplo: variação linear da altura $h(x)$. $\mathrm{Na}$ figura 2 mostra-se o perfil obtido pelo MEC para as duas malhas em comparação com o valor analítico correspondente. Os resultados apresentaram uma ótima concordância, com erros menores do que $0,5 \%$.

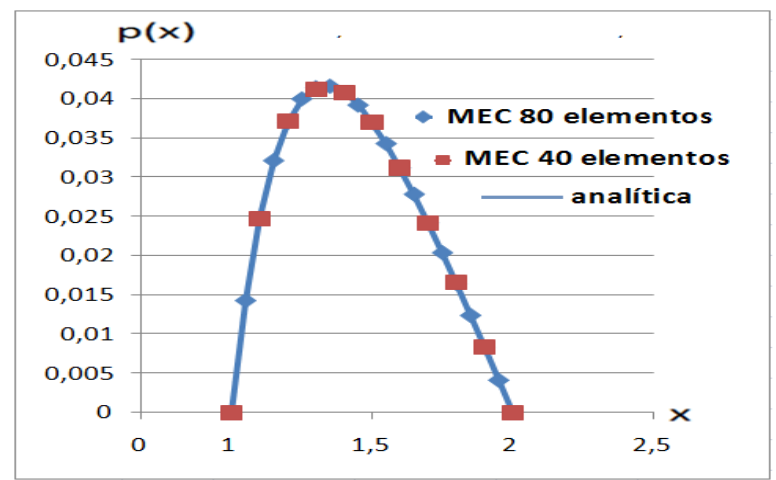

Figura 2: Solução numérica e solução analítica da pressão ao longo da direção do fluxo para variação linear da altura $h(x)$.

4.1 Segundo Exemplo: variação quadrática de $h(x)$. Neste caso, a ação de domínio se amplifica exponencialmente com relação ao exemplo anterior, de modo que se espera uma significativa redução da precisão do modelo numérico empregado. No entanto, os resultados desta simulação continuaram com muito boa precisão, conforme mostra a figura 3 .

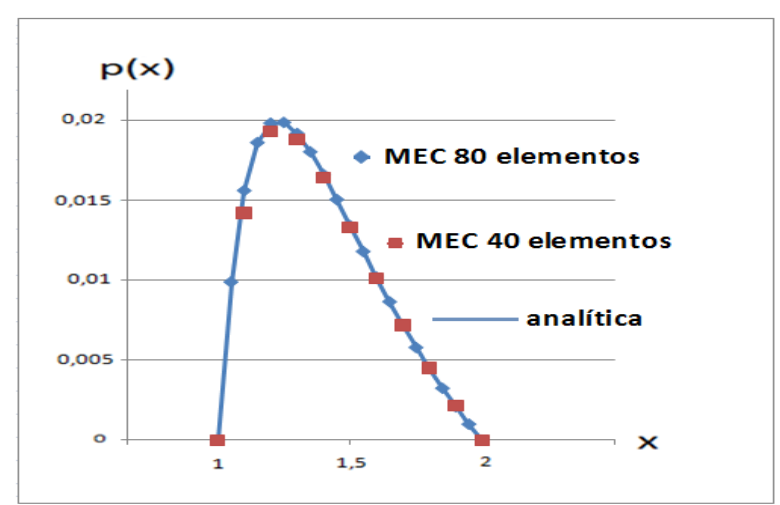

Figura 3: Solução numérica e solução analítica da pressão ao longo da direção do fluxo para variação quadrática da altura $\mathrm{h}(\mathrm{x})$.

\section{Referências}

[1] LOEFFLER, C. F.; CRUZ, A. L.; BULCÃO, A. Eng. Anal. Bound. Elements. v. 50, p.97-108, 2015.

[2] LOEFFLER, C. F.; PEREIRA, P. V. M.;BARCELOS, H. M.15 BETECH, p. 223-228, 2014.

[3] SPOTTS, M. F., Design of Machine Elements. New York: Prentice-Hall, 3를., 1961.

[4] BREBBIA, C. A. The Boundary Element Method for Engineering. London: Pentech Press, 1984.

[5] LOEFFLER, C. F.; MANSUR, W.J. Int, J. Num. Methods, Int. J. Num. Meth. Eng., v. 58, p. 1167-1186, 2003. 